\title{
Two unusual complications of neuroleptic malignant syndrome
}

\author{
A. Ahmad, C. A. Harrison', H. G. Davies²
}

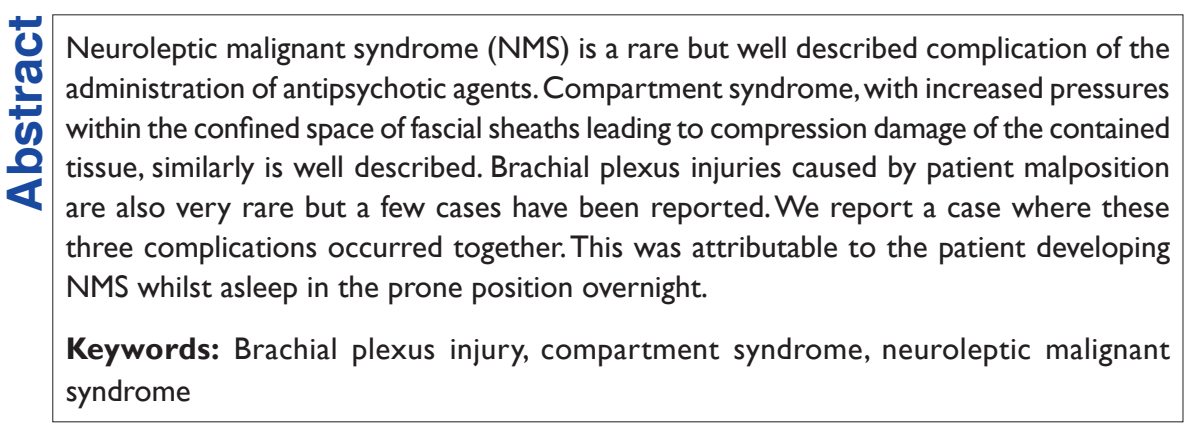

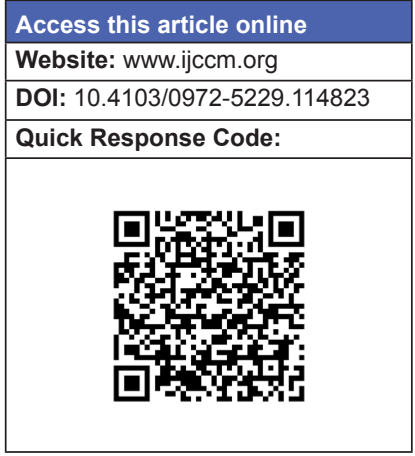

\section{Introduction}

We report a case of a 22 year old man with a history of schizophrenia who was admitted with neuroleptic malignant syndrome requiring mechanical ventilation. His admission was complicated by compartment syndrome and a brachial plexus injury in his right (dominant) arm. A review of the literature revealed that these are very rare complications of neuroleptic malignant syndrome that have not been reported together before.

\section{Case Report}

A 22-yr-old known schizophrenic was admitted to our hospital from a psychiatric unit where the nursing staff found him lying face down in bed, with his right arm bent beneath him. He was rigid and unresponsive. On admission, he was pyrexial, $39^{\circ}$, Blood pressure $70 / 40$, heart rate 168 beats $\mathrm{min}^{-1}$, respiratory rate 40 breaths min, ${ }^{[1]}$ Oxygen saturation was $100 \%$ on 15 litres

From:

Department of Anaesthesia and Intensive care, Morriston Hospital, Swansea,

${ }^{1}$ Department of Anaesthesia and Intensive care, ${ }^{2}$ Department of Trauma and

Orthopaedics, Nevill Hall Hospital, Abergavenny, United Kingdom

Correspondence:

Dr. Carol Harrison, Department of Anaesthesia, Nevill Hall Hospital, Abergavenny, NP7 7EG. United Kingdom.

E-mail: asifahmad2000@googlemail.com min $^{-1}$ via a Hudson mask and a Glasgow coma score of 6/15 (eyes four, best verbal response one, best motor response one). He had increased muscular tone and joint rigidity. There were multiple superficial skin lesions on his chest, medially on both knees and on his right forearm and thumb [Figure 1]. He had received a first dose of single depot of Flupentixol decanoate $80 \mathrm{mg}$ IM four days prior to admission. He was also on $300 \mathrm{mg}$ of clozapine daily. He had a history of alcohol and substance abuse and no known allergies.

The combination of muscular rigidity, hyperthermia

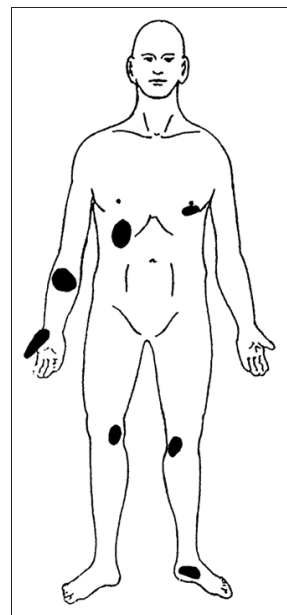

Figure I: Skin lesions 
and coma following an injection of an antipsychotic suggested the diagnosis of NMS. General anaesthesia was therefore induced in the emergency department with propofol and suxemethonium to facilitate intubation of the trachea. He also required initial fluid resuscitation with 6 litres of crystalloids and colloids.

His initial blood results were as follows:

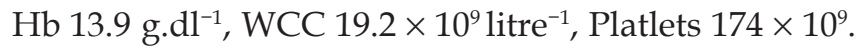
litre $^{-1}, \mathrm{Na}^{+} 148 \mathrm{mmol}$ litre $^{-1}, \mathrm{~K}^{+} 3.7 \mathrm{mmol}_{\text {litre }}{ }^{-1}$, HCO3 $19 \mathrm{mmol}_{\text {litre }}{ }^{-1}$, Urea $13.4 \mathrm{mmol}$ litre $^{-1}$, Creatinine 456 micromoles litre ${ }^{-1}$, Serum Creatine Kinase 31010 units litre $^{-1}$, Urine myoglobulin 961000 ng. $\mathrm{ml}^{-1}$. Arterial blood gas analysis: $\mathrm{Ph} 7.156, \mathrm{PO}_{2} 15.48 \mathrm{kPa}, \mathrm{PCO}_{2} 6.37 \mathrm{kPa}, \mathrm{BE}$ 12.2, HCO3 16.5 mmol litre ${ }^{-1}$.

He was transferred to Intensive Therapy Unit (ITU) where he was treated with supportive therapy including ventilation, paralysis, intravenous fluids, antipyretics, passive cooling and sedation. Multiple raised red lesions scattered over his body as pressure sores [Figure 1], increased swelling in the right forearm, tense and tender to palpation were apparent eight hours post admission.

The patient had developed compartment syndrome of the right forearm. The lesions on the volar aspect of right forearm, right chest, right thenar eminence and left nipple looked like 'kissing lesions'; the right forearm was compressed against the right chest and the right thenar eminence against the left nipple.

A fasciotomy with debridement of anterior compartments of right forearm and thenar components was performed. There was full thickness loss of skin, subcutaneous tissue and fascia and necrosis of the underlying volar forearm muscles extending into the deep flexor compartment including the median nerve.

The patient was discharged to the ward on day five. On day six he was taken back to the theatre for a superficial skin graft to the fasciotomy wound of right forearm, through a skin harvest from his thigh. He was discharged a week later to the psychiatric unit.

Further review a fortnight later revealed a persisting weakness of right elbow flexion and extension with wasting of supinator and deltoid muscle around his shoulder, suggesting a brachial plexus injury. An MRI of his cervical spine was normal. Nerve conduction studies confirmed a weakness of median and ulnar nerve roots.
Six months later, there was a gradually resolving weakness of deltoid and biceps muscle.

\section{Discussion}

Neuroleptic malignant syndrome (NMS) refers to combination of hyperthermia, rigidity and autonomic dysregulation that can occur as a complication of use of antipsychotic drugs. ${ }^{[1]}$ Amongst patients receiving neuroleptic agents, NMS's incidence is estimated as 0.07 to $2.2 \%{ }^{[2]}$ The estimated mortality is $11 \%{ }^{[3]}$

The pathogenesis of NMS although unclear, is based on the theories of central dopamine blockade and depletion in hypothalamus leading to abnormal central thermoregulation and primary skeletal muscle defect similar to malignant hyperthermia. NMS is associated with antipsychotics like thioxanthenes (flupentixol decanoate), phenothiazines, benzamides, butyrophenones and other agents such as clozapine and resperidones. ${ }^{[4]}$

The onset of NMS is not related to the duration of exposure to neuroleptics or to toxic overdose. ${ }^{[1]}$

The risk factors associated with NMS are patients with organic brain disease, learning difficulties, catatonia and chronic alcohol abuse. ${ }^{[5]}$

The diagnostic criteria for NMS consist of major criteria (fever, rigidity, elevated $\mathrm{CK}$ ) and minor criteria (tachycardia, abnormal blood pressure, tachypnoea, altered consciousness, diaphoresis and leucocytosis). ${ }^{[2]}$

The treatment of NMS depends on early recognition, hydration, nutrition and reduction of fever whilst secondary complications like hypoxia, acidosis and renal failure should be treated aggressively. Dopamine agonists such as bromocriptine and amantidine have also been used successfully.[2] The role of sodium dantrolene in NMS which inhibits calcium release from sarcoplasmic reticulum decreasing calcium for muscle contracture is debatable.

Compartment syndromes are characterised by increased tissue pressures within the confined space of fascial sheaths leading to increased pressure in the compartment, loss of integrity of microcirculation, oedema formation, muscle swelling and eventually compression of blood vessels and nerves. ${ }^{[6]}$ This in turn leads to tissue necrosis, which causes rhabdomyolysis, permanent functional impairment, renal failure and death. ${ }^{[6]}$ 
Treatment is based on early recognition and treatment. Necrosis occurs after six hours of ischaemia and fasciotomy should be performed within this time period.

Brachial plexus injuries are rare $(1.75 / 100,000$ population/year) with $60 \%$ caused by traction injuries, commonly involving root avulsions. ${ }^{[7]}$ In this case it is likely that the injury was caused by compression of the nerve roots. This is an uncommon cause of brachial plexus injury with an incidence of only $8.5 \%$ of all brachial plexus injuries. Brachial plexopathy has been reported in two patients after prone positioning in ITU and in one case after lying unconscious on the floor in diabetic coma. ${ }^{[7]}$ The brachial plexopathies caused by malposition previously reported have been transient. ${ }^{[7]}$ This is also true in our case.

The possibility of compartment syndrome in all patients with history of prolonged coma associated with malposition should be aggressively investigated and treated early in order to prevent long term functional impairment. This is even more imperative when the coma is due to NMS because of the additional complicating factors of markedly increased muscle tone and the inability of the patient to report their symptoms. Also, although a high serum creatine kinase and myoglobinuria are present in NMS other causes, such as compartment syndrome, should be excluded.

\section{References}

1. Adnet P, Lestavel P, Krivosic-Horber R. Neuroleptic malignant syndrome. Br J Anaesth 2000;85:129-35.

2. Stotz M, Thümmler D, Schürch M, Renggli JC, Urwyler A, Pargger H. Fulminant neuroleptic malignant syndrome after perioperative withdrawal of anti Parkinsonian medication. Br J Anaesth 2004;93:868-71.

3. Shalev A, Hermesh H, Munitz H. Mortality from neuroleptic malignant syndrome. J Clin Psychiatry 1989;50:18-25.

4. Hassan S, Buckley P. Novel antipsychotics and the neuroleptic malignant syndrome: A review and critique. Am J Psych 1998;155:1113-6.

5. Guerra R. Sympathoadrenal hyperactivity and the aetiology of neuroleptic malignant syndrome. Am J Psych 1999;156:169-80.

6. O'Donnell CJ, Beck DH, Taylor BL, Smith GB. Upper limb compartment syndromes: A complication of malignant hyperthermia in a patient with ill-defined myopathy. Br J Anaesth 1995;74:343-4.

7. Goettler CE, Pryor JP, Reilly PM. Brachial plexopathy after prone positioning. Crit Care 2002;6:540-42.

How to cite this article: Ahmad A, Harrison CA, Davies HG. Two unusual complications of neuroleptic malignant syndrome. Indian J Crit Care Med 2013;17:116-8.

Source of Support: Nil, Conflict of Interest: None declared.

\section{Dispatch and return notification by E-mail}

The journal now sends email notification to its members on dispatch of a print issue. The notification is sent to those members who have provided their email address to the association/journal office. The email alerts you about an outdated address and return of issue due to incomplete/incorrect address.

If you wish to receive such email notification, please send your email along with the membership number and full mailing address to the editorial office by email. 